\title{
Malocclusion and dental appearance in underprivileged Brazilian adolescents
}

\author{
Armando Koichiro KAIEDA(a) \\ Jaqueline Vilela BULGARELI(a) \\ Inara Pereira da CUNHA ${ }^{(a)}$ \\ Sílvia Amélia Scudeler \\ VEDOVELLO(b) iD \\ Luciane Miranda GUERRA(a) iD \\ Gláucia Maria Bovi \\ AMBROSANO(a) iD \\ Antonio Carlos PEREIRA(a) iD \\ Luiz Renato PARANHOS(c) \\ Karine Laura CORTELLAZZI (a)

\footnotetext{
(a) Universidade Estadual de Campinas - Unicamp, Piracicaba Dental School, Department of Community Dentistry, Piracicaba, SP, Brazil.

(b) Centro Universitário Hermínio Ometto Uniararas, Dentistry Course, Post-Graduate in Orthodontics, Mogi Guaçu, SP, Brazil.

(c) Universidade Federal de Uberlândia UFU, Department of Preventive and Social
} \\ Dentistry, Uberlândia, MG, Brazil.
}

Declaration of Interests: The authors certify that they have no commercial or associative interest that represents a conflict of interest in connection with the manuscript.

\section{Corresponding Author:}

Karine Laura Cortellazzi

E-mail: karinecortellazzi@gmail.com

hitps://doi.org/10.1590/1807-3107bor-2019.vol33.0014

Submitted: August 23, 2018

Accepted for publication: November 11, 2018

Last revision: January 15, 2019
Abstract: Satisfaction with dental aesthetics is a subjective indicator used in epidemiological studies and is related to health behaviours. Little is known about the factors that influence this indicator, particularly among adolescents who live in a situation of social vulnerability. The aim of this study was to investigate the relationship between malocclusion and dental appearance in underprivileged Brazilian adolescents. This analytical cross-sectional study was conducted in Piracicaba, Brazil, and evaluated 884 adolescents from 13 to 19 years of age. The dependent variable was satisfaction with dental appearance, and the independent variables were classified as individual (components of the Dental Aesthetic Index - DAI, sex and age) and contextual (social exclusion index). For statistical analysis, multilevel regression models were estimated. The individual variables were considered Level 1 , and the contextual variable was considered Level 2, with a level of significance of $5 \%$. The mean age of the adolescents was 15.3 years. Female adolescents more frequently affirmed that they were satisfied with their dental appearance than did male individuals. There was an increase in dissatisfaction with oral health with the increase in anterior maxillary overjet, midline diastema, larger anterior irregularity in the maxilla, larger anterior irregularity in the mandible, anterior open bite and antero-posterior molar relation. Satisfaction with dental appearance was associated with individual factors such as sex and DAI components.

Keywords: Malocclusion; Adolescent; Esthetics, Dental.

\section{Introduction}

One of the motivating factors of orthodontic treatment is the search for improvement in facial appearance. ${ }^{1}$ However, there are still differences between the individual perception of aesthetics and the need for treatment assessed by a professional. ${ }^{2,3,4}$ Perception is a subjective dimension that individuals have of themselves and different aspects of life ${ }^{5}$ and may be influenced by individual factors such as the presence of changes in occlusion, sex and age, and / or social factors. ${ }^{6}$

From this aspect, malocclusion has been considered a cause of various functional problems and aesthetic and social relations, ${ }^{7}$ causing a negative impact on the quality of life of adolescents. ${ }^{8,9,10,11}$ When aesthetic factors are involved, individuals are subjected to various judgements regarding physical attractiveness and social acceptance. ${ }^{12,13}$ The aesthetic impairment 
caused by malocclusion arises from changes in the smile. Moreover, it is noteworthy that there are some types of malocclusion that have more influence on the satisfaction with dental appearance. ${ }^{14}$

Other factors that contribute to the perception of dental aesthetics are of a sociodemographic nature; among them are family income ${ }^{15,16}$ and sex. ${ }^{5,17}$ Different levels of poverty are related to social vulnerability and are responsible for a higher number of risk factors that lead to individuals becoming ill.

Vulnerability can be measured by the social exclusion index (SEI) ${ }_{1}^{18}$ which summarizes the situation of the municipality in which certain people live and considers several indicators related to social determinants of health, such as the formal employment rate, literacy and local violence.

The Dental Aesthetic Index (DAI) is a numeric index with different criteria for evaluating malocclusion. The index links clinical and aesthetic components that reflect physical and aesthetic patterns of occlusion, including the patients' perceptions. ${ }^{19}$ However, the association between the level of poverty and the impact of tooth position on changes in the perception of dental appearance has not yet been fully understood. Thus, it is necessary to develop studies that contribute to the analysis of risk groups and orthodontic treatment planning in public services.

Given the global demand for orthodontic treatment, there is a need to develop methods to assess treatment according to the severity of malocclusion or even to understand the reasons that determine the demand for services.

Therefore, understanding the factors related to the perception of dental aesthetics among vulnerable adolescents could contribute to the development of strategies aimed at increasing access to dental services.

Thus, the aim of this study was to investigate the relationship between malocclusion and selfperception of dental appearance in underprivileged Brazilian adolescents.

\section{Methodology}

Ethical criteria: This study was approved by the Research Ethics Committee of of FOP-UNICAMP, in accordance with resolution 466/12 of the National
Health Council, Ministry of Health, under Protocol No. No.027/2011, and in compliance with the ethical and legal criteria of the research.

\section{Type of study and location}

This cross-sectional study was conducted in the municipal schools in the city of municipality of Piracicaba, State of São Paulo, Brazil, and involved adolescents from 13 to 19 years of age who had been receiving primary health care from a Family Health Unit (PHC-FH) between July 2014 and March 2015. The participants were enrolled in public schools (located in the territories covered by these PHC-FH units) and in the PHC-FC units. Social exclusion occurred to the greatest extent in these regions in the municipality.

\section{Study site}

Piracicaba has an estimated population of 368,843 inhabitants and a human development index of 0.84 . The city has a total of 12,539 adolescents in the age range of 13 to 19 years. According to the data from the Municipal Secretary for Health, there was a total of 34 PHC-FHs, among which were 12 units with primary dental care teams. On average, 320 adolescents between the ages of 13 and 19 years were enrolled in each of the PHC-FHs, totalling approximately 11,000 individuals. It is important to emphasize that the PHC units are distributed in the less favoured socioeconomic regions of the municipality. All public high schools $(n=21)$ in the territorial areas covered by the PHCs were included in the study. In the 34 PHC units, the terms of free and informed consent to participate in the study were given to the community health agents for use during home visits. These agents also previously made the appointments for the time and day for participants to appear at the units. At the schools, the terms of informed consent were given to the teachers, who distributed them to the selected schoolchildren to subsequently obtain the parents' or guardians' authorization.

\section{Sample size}

The sample size was calculated considering a confidence interval of $95 \%$, a test power of 0.80 , an odds ratio $=1.4,60 \%$ of the individuals having a high level of dissatisfaction and low treatment needs, and the 
procurement of a sample of 1,258 randomly selected individuals aged 13-19 years. Of these individuals, 374 were eliminated due to the exclusion criteria. A total of 884 adolescents were examined; 221 individuals were examined at the 34 PHC-HF units, and 663 were examined at the 21 public high schools. Of the total 884 adolescents, 850 (96.1\%) resided in suburbs with the worst social exclusion indices (negative indices).

\section{Inclusion and exclusion criteria}

Adolescents who had systemic diseases, significant facial asymmetry and orthodontic treatment before inclusion in the study were excluded.

\section{Clinical examination}

The exams were performed under artificial light at the schools and at the PHC-FHs by two previously trained examiners who were assisted by two note-takers. The standardized clinical record chart was used, and previous tooth brushing was performed under the supervision of an oral health technician. For each exam, a periodontal probe and a plane dental mirror No. 5 were used. ${ }^{20}$ The clinical examination for malocclusion was performed according to the DAI index, which is a continuous scale to assess the severity levels to prioritize the treatment need. ${ }^{19}$

\section{Calibration}

Before the study began, a calibration process was conducted between the examiners to obtain acceptable consistency for all the clinical conditions. The theoretical-practical activities of the training and calibration exercises consisted of a total of 7 periods as follows: 1 theoretical period lasting 4 hours; 4 clinical training sessions of 4 hours each (a total of 16 h), and 2 calibration exercises lasting 4 hours (a total of $8 \mathrm{~h}$ ). The training stage consisted of a theoretical discussion followed by a practical stage, during which the examiners evaluated 12 adolescents per period. During training and calibration, the interand intra-examiner agreement was estimated by the intraclass correlation coefficient (ICC) for the dental aesthetic index (DAI) and the weighted kappa for their components, with an acceptable limit value greater than 0.80 .

\section{Study instruments and variables}

The dependent variable of the study was selfperception of oral health ("Are you satisfied with the appearance of your teeth?") classified as high satisfaction, satisfaction, low satisfaction and high dissatisfaction according to the questionnaire used. ${ }^{21}$

The independent variables were classified into individual and contextual types. The clinical (components of the dental aesthetic index, DAI) and demographic characteristics (age, sex) were considered individual variables (Level 1). In Level 2 (contextual variable), the social exclusion index (SEI) was analysed with reference to the characteristics of the suburbs.

The DAI is an orthodontic index based on socially defined aesthetic standards. ${ }^{19}$ The clinical and aesthetic components combine a single result of the physical and aesthetic aspects of occlusion. ${ }^{22,23} \mathrm{DAI}$ is a numerical index that evaluates the occlusal characteristic selected according to the potential of causing psychosocial incapacity. It includes parameters of dentofacial anomalies related to the following clinical and aesthetic aspects: the number of visibly absent teeth, anterior crowding, anterior spacing, midline diastema, maxillary anterior misalignment, maxillary anterior horizontal overlap, mandibular anterior horizontal overlap, anterior open bite, antero-posterior molar relation and posterior crossbite. In general, the DAI index score is analysed by means of the sum of scores of each characteristic evaluated added to a constant value. This sum leads to a classification that identifies the orthodontic treatment need of individuals determined by the severity of the occlusal pathologies present. ${ }^{22}$ In the present study, for the purposes of analysis, the index was not calculated mathematically, but its components were used in a separate manner with the purpose of verifying which of them were related to the self-perception of dental appearance.

The SEI of the 36 suburbs where the adolescents resided was collected from the Piracicaba Research and Planning Institute and the Municipal Secretary for Social Development Performances. ${ }^{18}$ The purpose was to quantify some of the attributes of social inequalities between the suburbs, ranging from -1 (most vulnerable) to 1 (least vulnerable). Other items 
of information with reference to the suburbs where the adolescents resided (total number of residents per suburb, literacy rate, \% of home ownership, \% domestic sewage available, \% garbage collected, \% with income of up to 0.5 minimum wage, $\%$ with income from 0.5 to 1 minimum wage, $\%$ with income higher than 1 minimum wage and \% without monthly income) were obtained from the Brazilian Institute of Geography and Statistics.

\section{Data analysis}

The multilevel regression models were estimated by the PROC GLIMMIX - "Generalized Linear Models-Mixed"- procedures using SAS 9.2 statistical software. In the analysis, the individual variables were considered Level 1, and the variable SEI was considered Level 2. The statistical significance was evaluated at a level of significance of $5 \%$.

Initially, a model was estimated with only the intercept to study the proportion of variance due to the suburbs in relation to the individuals. This model served as the basis for evaluating the reduction in the variance of the other models studied (Model 1). The individual variables (Model 2) were then tested, and later, the variable SEI was included (Model 3). Adjustment of the model was evaluated by the -2 Res Log Likelihood (the lower the value is, the better is the fit of the model).

\section{Results}

The final sample was composed of 884 adolescents with a mean age of 15.3 years ( $\mathrm{SD}=1.01)$. Of these, 469 (55.97\%) participants were female, and 413 (44.03\%) participants were male.

Table 1 shows the frequency of the response variable with regards to self-perception of oral health. The majority of adolescents answered that they had low satisfaction with the appearance of their teeth, and $35.6 \%$ and $30.1 \%$ were highly dissatisfied.

Table 2 shows the distribution, median, minimum and maximum values of the SEI and DAI components.

The multilevel model for self-perception of oral health may be observed in Table 3. When the individual variables were included in Model 2, the reduction in the - 2 Res Log Likelihood was approximately 3.3\%.
The variable social exclusion index was not significant in the model $(p=0.3886)$.

Therefore, for this population, the variation in self-perception of oral health was explained by the variables related to the individuals (age, sex, and DAI) and not the suburbs (SEI). Considering the level of significance of 5\%, in Model 2, it was observed that the randomly selected participants of the female sex presented a higher satisfaction with oral health in comparison with that of the male sex $(p<0.0001)$. There was an increase in dissatisfaction with oral health with the increase in anterior maxillary overjet ( $p=0.0561)$, midline diastema $(p=0.0008)$, largest anterior irregularity in the maxilla ( $p<0.0001)$, largest anterior irregularity in the mandible $(\mathrm{p}=0.0209)$, anterior open bite $(p=0.0118)$ and antero-posterior molar relation $(\mathrm{p}=0.0327)($ Table 3$)$.

\section{Discussion}

This study revealed that $30 \%$ of adolescents were dissatisfied with their own dental aesthetics. This dissatisfaction with dental appearance showed a statistical relationship with the adolescents' sex and with some of the occlusal components, such as the following: median maxillary diastema, mandibular irregularity, anterior open bite and the anteroposterior molar relation, evaluated by the Dental Aesthetic Index.

Malocclusion with its different levels of severity is capable of compromising not only the aesthetic appearance of individuals ${ }^{23,24}$ but also their emotional stability, since compromised smile aesthetics can decrease their self-esteem ${ }^{25}$ and social life. ${ }^{13}$ Moreover, adolescents with malocclusion are perceived as being less popular, bad at sports and incapable of being leaders, with less chance of finding a job. ${ }^{26}$

Table 1. Distribution of perception on dental appearance.

\begin{tabular}{lcc}
\hline Self-perception of oral health & $\mathrm{n}$ & $\%$ \\
\hline High satisfaction & 63 & 7.1 \\
Satisfaction & 240 & 27.2 \\
Low satisfaction & 314 & 35.6 \\
High dissatisfaction & 265 & 30.1 \\
\hline
\end{tabular}


Table 2. Mean, median, minimum and maximum values of SEI and DAI components.

\begin{tabular}{|c|c|c|c|}
\hline Variable & Mean & Minimum & Maximum \\
\hline SEI & -0.49 & -1.00 & 0.79 \\
\hline DAl components & Median & Minimum & Maximum \\
\hline $\begin{array}{l}\text { Number of missing visible teeth (incisors, canines and premolars in the } \\
\text { maxillary and mandibular arches) }\end{array}$ & 0.00 & 0.00 & 3.00 \\
\hline Anterior maxillary overjet & 3.00 & 0.00 & 14.00 \\
\hline Anterior mandibular overjet & 0.00 & 0.00 & 4.00 \\
\hline Incisal segment crowding & 1.00 & 0.00 & 2.00 \\
\hline Incisal segment spacing & 0.00 & 0.00 & 2.00 \\
\hline Midline diastema & 0.00 & 0.00 & 5.00 \\
\hline Largest anterior irregularity on the maxilla & 0.00 & 0.00 & 8.00 \\
\hline Largest anterior irregularity on the mandible & 1.00 & 0.00 & 7.00 \\
\hline Anterior open bite & 0.00 & 0.00 & 9.00 \\
\hline Antero-posterior molar relation & 0.00 & 0.00 & 2.00 \\
\hline
\end{tabular}

SEI: Social exclusion index; DAI: Dental aesthetic index.

Table 3. Multilevel model for perception of dental appearance.

\begin{tabular}{|c|c|c|c|c|c|c|c|c|c|}
\hline \multirow{2}{*}{ Variable } & \multicolumn{3}{|c|}{ Model 1} & \multicolumn{3}{|c|}{ Model 2} & \multicolumn{3}{|c|}{ Model 3} \\
\hline & Estimate* & \#SE & $\mathrm{p}$-value & Estimate & SE & $\mathrm{p}$-value & Estimate & SE & $\mathrm{p}$-value \\
\hline Intercept & 2.88 & 0.03 & $<0.0001$ & 2.86 & 0.03 & $<0.0001$ & 2.86 & 0.03 & $<0.0001$ \\
\hline \multicolumn{10}{|l|}{ Individual level } \\
\hline Sex $($ Ref $=$ Male $)$ & & & & -0.23 & 0.06 & $<0.0001$ & -0.23 & 0.06 & $<0.0001$ \\
\hline Anterior maxillary overjet & & & & 0.03 & 0.02 & 0.0561 & 0.03 & 0.02 & 0.0561 \\
\hline Midline diastema & & & & 0.15 & 0.04 & 0.0008 & 0.15 & 0.04 & 0.0008 \\
\hline Largest anterior irregularity on the maxilla & & & & 0.12 & 0.02 & $<0.001$ & 0.12 & 0.02 & $<0.001$ \\
\hline Largest anterior irregularity on the mandible & & & & 0.07 & 0.03 & 0.0209 & 0.07 & 0.03 & 0.0209 \\
\hline Anterior open bite & & & & 0.11 & 0.04 & 0.0118 & 0.11 & 0.04 & 0.0118 \\
\hline Antero-posterior molar relation & & & & 0.10 & 0.01 & 0.0327 & 0.10 & 0.01 & 0.0327 \\
\hline \multicolumn{10}{|l|}{ Suburb level } \\
\hline Social Exclusion Index (SEI) & & & & & & & $\$$ ns & & \\
\hline 2 Res Log Likelihood & \multicolumn{3}{|c|}{2359.74} & \multicolumn{3}{|c|}{2281.40} & \multicolumn{3}{|c|}{2281.40} \\
\hline
\end{tabular}

*Parameter estimate; \# Standard error of parameter estimate; ${ }^{5}$ Not significant ( $\left.p=0.3886\right)$.

Furthermore, different types of malocclusion may produce different functional changes. In this study, some DAI components influenced the dissatisfaction with appearance. The presence of an open bite, for example, was identified as a risk indicator of speech capability, and mandibular overjet and the molar ratio were identified as risk factors for the expression of capacity and chewing, respectively. ${ }^{14}$

However, it is important to understand other factors that may be involved in self-perception of dental appearance, especially with regards to the expectation of orthodontic treatment. Studies have shown that there were differences between the perceived need of adolescents and the treatment need indicated by professionals, ${ }^{2,3,4}$ which could compromise the orthodontic planning and treatment satisfaction.

Orthodontic treatment was included in the list of specialized therapeutic alternatives offered by the health system, enabling the diagnosis and treatment of malocclusions with two main objectives: to assess the need and the priority treatment of individuals and to obtain information to enable proper allocation of the necessary resources for providing the population with orthodontic treatment. Considering the results of this study, when the DAI was used in conjunction with selfperception of oral health, it could be considered a good epidemiological tool to define the priority of orthodontic care for the purpose of allocating public health resources. 
Another factor that influenced the perception was the adolescents' sex. More of the girls said they were satisfied with their dental appearance than boys, unlike other studies that reported that more boys were satisfied, ${ }^{5,27}$ and there was no difference between the sexes. ${ }^{13,28}$ The most plausible explanation for this in this study is that girls are more careful about their oral health and more frequently seek dental services than boys. ${ }^{29,30}$ This is related to the concern about aesthetic dentistry and oral care. ${ }^{31}$ Therefore, this attention to the teeth could influence the perception of aesthetics.

It seems reasonable to say that by means of the severity of malocclusion, it could be possible to predict the perception and the need for orthodontic treatment among adolescents. The present study demonstrated that dissatisfaction was associated with poor positioning of the teeth, which showed that even in vulnerable segments of Brazilian society, the attributes of beauty were a stunning and remarkable characteristic of western culture. ${ }^{31}$ Previous studies conducted in the country also corroborated this finding. ${ }^{17,32}$

Previous research has shown the relationship of malocclusion with socioeconomic factors. ${ }^{10,11,33}$ This is because the social condition is related to the access to services, ${ }^{34}$ exposure to risk factors and health behaviours. ${ }^{16}$ However, other mechanisms are involved in health and social support and coping mechanisms of adverse situations, which go beyond social status. ${ }^{35}$

Brazil has a public health system, which is organized into low, medium and high complexities. Relative to oral health, cases of low complexity are coordinated by an oral health team in primary care, which is not limited to clinical care but includes actions of prevention and health education.

As a result, this suggests that in addition to prevention and health education, the oral health teams included in primary care must identify and refer the cases of malocclusion and seek to strengthen the social support networks in the community, thereby transposing the consequences of health determinants embedded in the setup of vulnerable segments.

One of the differentials of this study was the use of a multilevel model in the analysis of factors related to dental appearance in adolescents. Multilevel analysis is normally the method of choice, working with two different levels of effects (individual and contextual) and how they interrelate and produce oral health outcomes. Variables related to the socioeconomic level generally play an important role in the multilevel model because the increase in monthly family income increases the quality of life and aspects related to oral health. ${ }^{36}$

In addition to investigating the factors that influenced the self-perception of dental appearance in underprivileged Brazilian adolescents, in a specific approach to the population of underprivileged adolescents, the present study endeavoured to establish the variables that identified environments of vulnerability. Most of the studies have been conducted on an individual basis; there are few that have evaluated the impact of territorial characteristics on oral health, especially considering the underprivileged population.

A limitation of this study was its cross-sectional design; this type of study does not allow examination of the causal relationship between the variables investigated and the outcome. The contextual data were obtained from government sources, which are subject to problems of under- or over-notification, processing errors, not updating indicators, and other issues. However, this study has strengths, such as the random selection of adolescents, expected response rate, the robust calibration process of oral health examiners and the use of acceptable measures of the DAI.

Therefore, the authors suggest continuity of the study by means of new study designs that may provide answers that are broader in scope to the complex questions involving self-perception of oral health in adolescents. From this aspect, it is also opportune to adopt qualitative methods of study, which seek to go more deeply into the meanings of the adolescents' occlusal problems and their perception of satisfaction with their teeth.

\section{Conclusion}

Satisfaction with dental appearance was associated with individual factors such as sex and components of the DAI.

\section{Acknowledgements}

The authors thank the National Counsel of Technological and Scientific Development-CNPq for their support in the research [grant numbers 141654/2013-9]. 
Kaieda AK, Bulgareli JV, Cunha IP, Vedovello SAS, Guerra LM, Ambrosano GMB et al.

\section{References}

1. Perillo L, Esposito M, Caprioglio A, Attanasio S, Santini AC, Carotenuto M. Orthodontic treatment need for adolescents in the Campania region: the malocclusion impact on self-concept. Patient Prefer Adherence. 2014 Mar;8:353-9. https://doi.org/10.2147/PPA.S58971

2. Yin L, Jiang M, Chen W, Smales RJ, Wang Q, Tang L. Differences in facial profile and dental esthetic perceptions between young adults and orthodontists. Am J Orthod Dentofacial Orthop. 2014 Jun;145(6):750-6. https://doi.org/10.1016/i.ajodo.2014.01.021

3. Eslami N, Omidkhoda M, Shafaee H, Mozhdehifard M. Comparison of esthetics perception and satisfaction of facial profile among male adolescents and adults with different profiles. J Orthod Sci. 2016 Apr-Jun;5(2):47-51. https://doi.org/10.4103/2278-0203.179406

4. Silva LF, Thomaz EB, Freitas HV, Ribeiro CC, Pereira AL, Alves CM. Self-perceived need for dental treatment and related factors. A crosssectional population-based study. Braz Oral Res. 2016;30(1):e0055. https://doi.org/10.1590/1807-3107BOR-2016.vol30.0055

5. Di Blasi M, Cavani P, Pavia L, Lo Baido R, La Grutta S, Schimmenti A. The relationship between self $\square$ lmage and social anxiety in adolescence. Child Adolesc Ment Health. 2015;20(2):74-80. https://doi.org/10.1111/camh.12071

6. Krisdapong S, Prasertsom P, Rattanarangsima K, Sheiham A. Associations between perceived needs for dental treatment, oral healthrelated quality of life and oral diseases in school-aged Thai children. Community Dent Oral Epidemiol. 2014 Aug;42(4):323-32. https://doi.org/10.1111/cdoe.12092

7. Choi SH, Kim Bl, Cha JY, Hwang CJ. Impact of malocclusion and common oral diseases on oral health-related quality of life in young adults. Am J Orthod Dentofacial Orthop. 2015 May;147(5):587-95. https://doi.org/10.1016/i.ajodo.2014.12.025

8. Dimberg L, Arnrup K, Bondemark L. The impact of malocclusion on the quality of life among children and adolescents: a systematic review of quantitative studies. Eur J Orthod. 2015 Jun;37(3):238-47. https://doi.org/10.1093/ejo/cju046

9. Marques LS, Ramos-Jorge ML, Paiva SM, Pordeus IA. Malocclusion: esthetic impact and quality of life among Brazilian schoolchildren. Am J Orthod Dentofacial Orthop. 2006 Mar;129(3):424-7. https://doi.org/10.1016/i.ajodo.2005.11.003

10. Vedovello SA, Ambrosano GM, Pereira AC, Valdrighi HC, Filho MV, Meneghim MC. Association between malocclusion and the contextual factors of quality of life and socioeconomic status. Am J Orthod Dentofacial Orthop. 2016 Jul;150(1):58-63. https://doi.org/10.1016/j.ajodo.2015.12.022

11. Benson PE, Da'as T, Johal A, Mandall NA, Williams AC, Baker SR, et al. Relationships between dental appearance, self-esteem, socio-economic status, and oral health-related quality of life in UK schoolchildren: A 3-year cohort study. Eur J Orthod. 2015 Oct;37(5):481-90. https://doi.org/10.1093/ejo/cju076

12. Jung MH. Evaluation of the effects of malocclusion and orthodontic treatment on self-esteem in an adolescent population. Am J Orthod Dentofacial Orthop. 2010 Aug;138(2):160-6. https://doi.org/10.1016/i.ajodo.2008.08.040

13. Olsen JA, Inglehart MR. Malocclusions and perceptions of attractiveness, intelligence, and personality, and behavioral intentions. Am J Orthod Dentofacial Orthop. 2011 Nov;140(5):669-79. https://doi.org/10.1016/j.ajodo.2011.02.025

14. Peres SH, Goya S, Cortellazzi KL, Ambrosano GM, Meneghim MC, Pereira AC. Self-perception and malocclusion and their relation to oral appearance and function. Cien Saude Colet. 2011 Oct;16(10):4059-66. https://doi.org/10.1590/S1413-81232011001100011

15. Gabardo MC, Moysés SJ, Moysés ST, Olandoski M, Olinto MT, Pattussi MP. Multilevel analysis of self-perception in oral health and associated factors in Southern Brazilian adults: a cross-sectional study. Cad Saude Publica. 2015 Jan;31(1):49-59. https://doi.org/10.1590/0102-311X00037814

16. Silveira MF, Freire RS, Nepomuceno MO, Martins AM, Marcopito LF. Severity of malocclusion in adolescents: populational-based study in the north of Minas Gerais, Brazil. Rev Saude Publica. 2016;50(0):11. https://doi.org/10.1590/S1518-8787.2016050005861

17. Peres KG, Barros AJ, Anselmi L, Peres MA, Barros FC. Does malocclusion influence the adolescent's satisfaction with appearance? A cross-sectional study nested in a Brazilian birth cohort. Community Dent Oral Epidemiol. 2008 Apr;36(2):137-43. https://doi.org/10.1111/i.1600-0528.2007.00382.x

18. Instituto de Pesquisas e Planejamento de Piracicaba - IPPLAP. [webpage]. Piracicaba: Instituto de Pesquisas e Planejamento de Piracicaba; 2017. [Accessed 2017 Nov 19]. Available from: http: //www.ipplap

19. Jenny J, Cons CN. Guidelines for using the DAI: a supplement to DAI: the Dental Aesthetic Index College of Dentistry. lowa City: University of lowa 1988.

20. World Health Organization - WHO. WHO Oral health surveys: basic methods. Geneva: World Health Organization;.1987.

21. Goes PS, Watt R, Hardy RG, Sheiham A. The prevalence and severity of dental pain in 14-15 year old Brazilian schoolchildren. Community Dent Health. 2007 Dec;24(4):217-24

22. Jenny J, Cons NC, Kohout FJ, Jakobsen J. Differences in need for orthodontic treatment between Native Americans and the general population based on DAl scores. J Public Health Dent. 1991;51(4):234-8. https://doi.org/10.1111/j.1752-7325.1991.tb02221.x

23. Paula DF Jr, Silva ET, Campos AC, Nuñez MO, Leles CR. Effect of anterior teeth display during smiling on the self-perceived impacts of malocclusion in adolescents. Angle Orthod. 2011 May;81(3):540-5. https://doi.org/10.2319/051710-263.1 
Malocclusion and dental appearance in underprivileged Brazilian adolescents

24. Tessarollo FR, Feldens CA, Closs LQ. The impact of malocclusion on adolescents' dissatisfaction with dental appearance and oral functions. Angle Orthod. 2012 May;82(3):403-9.https://doi.org/10.2319/031911-195.1

25. Gavric A, Mirceta D, Jakobovic M, Pavlic A, Zrinski MT, Spali S. Craniodentofacial characteristics, dental esthetics-related quality of life, and self-esteem. Am J Orthod Dentofacial Orthop. 2015 Jun;147(6):711-8.https://doi.org/10.1016/j.ajodo.2015.01.027

26. Pithon MM, Nascimento CC, Barbosa GC, Coqueiro RS. Do dental esthetics have any influence on finding a job? Am J Orthod Dentofacial Orthop. 2014 Oct;146(4):423-9. https://doi.org/10.1016/i.ajodo.2014.07.001

27. Grecu A, Ciutrila I, Lasserre JF, Colosi H, Culic B, Dudea D. Selfperception in dental aesthetics-A study in two ethnic groups. J Psychosom Res. 2014;76(6):503-4. https://doi.org/10.1016/i.jpsychores.2014.03.045

28. Onyeaso CO, Sanu OO. Perception of personal dental appearance in Nigerian adolescents. Am J Orthod Dentofacial Orthop. 2005 Jun;127(6):700-6. https://doi.org/10.1016/i.ajodo.2003.12.028

29. Peres MA, Peres KG, de Barros AJ, Victora CG. The relation between family socioeconomic trajectories from childhood to adolescence and dental caries and associated oral behaviours. J Epidemiol Community Health. 2007 Feb;61(2):141-5. https://doi.org/10.1136/jech.2005.044818

30. Brizon VS, Cortellazzi KL, Vazquez FL, Ambrosano GM, Pereira AC, Gomes VE, et al. [Individual and contextual factors associated with malocclusion in Brazilian children]. Rev Saude Publica. 2013 Dec;47 Suppl 3:118-28. Portuguese. https://doi.org/10.1590/S0034-8910.2013047004426

31. Xiao-Ting L, Tang Y, Huang XL, Wan H, Chen YX. Factors influencing subjective orthodontic treatment need and culture-related differences among Chinese natives and foreign inhabitants. Int J Oral Sci. 2010 Sep;2(3):149-57. https://doi.org/10.4248/IJOS10050

32. Moura C, Cavalcanti AL, Gusmão ES, Soares RS, Moura FT, Santillo PM. Negative self-perception of smile associated with malocclusions among Brazilian adolescents. Eur J Orthod. 2013 Aug;35(4):483-90. https://doi.org/10.1093/ejo/cjs022

33. Normando TS, Barroso RF, Normando D. Influence of the socioeconomic status on the prevalence of malocclusion in the primary dentition. Dental Press J Orthod. 2015 Jan-Feb;20(1):74-8. https://doi.org/10.1590/2176-9451.20.1.074-078.oar

34. Cheema J, Sabbah W. Inequalities in preventive and restorative dental services in England, Wales and Northern Ireland. Br Dent J. 2016 Sep;221(5):235-9. https://doi.org/10.1038/sj.bdj.2016.641

35. Taylor SE, Seeman TE. Psychosocial resources and the SES-health relationship. Ann N Y Acad Sci. 1999;896(1):210-25. https://doi.org/10.1111/j.1749-6632.1999.tb08117.x

36. Vazquez FL, Cortellazzi KL, Kaieda AK, Guerra LM, Ambrosano GM, Tagliaferro EP, et al. Quality of life and socio-dental impact among underprivileged Brazilian adolescents. Qual Life Res. 2015 Mar;24(3):661-9. https://doi.org/10.1007/s11136-014-0795-4 